\title{
Increasing Sleep Health Awareness in Pediatric Settings: Creating Opportunities for Generating a More Sleep-literate Health Care Workforce
}

\author{
Sarah Blunden ${ }^{1}$ (D) Perran Boran ${ }^{2}$ (D) \\ ${ }^{1}$ Central Queensland University, Appleton Institute of Behavioural Science, Adelaide, Australia \\ ${ }^{2}$ Marmara University, School of Medicine, Istanbul, Turkey
}

ORCID ID: S.B. 0000-0002-5026-1992; P.B. 0000-0002-9885-7656

Citation/Atf: Blunden S, Boran P. Increasing sleep health awareness in pediatric settings: creating opportunities for generating a more sleep-literate health care workforce. Çocuk Dergisi - Journal of Child 2021;21(3):293-296. https://doi.org/10.26650/jchild.2021.1003751

\section{ABSTRACT}

Sleep health is one of the major contributors to mental and physical health and as such should be an essential component of informed community and policy conversations around health. Non-physiological sleep problems are commonly referred to as Behavioral Sleep Problems (BSP) and include difficulties initiating sleep alone, bedtime resistance, difficulties re-initiating sleep after normally occurring overnight waking (requiring parental assistance to re-settle) and/or early morning wakening. BSP affects up to $40 \%$ of children under two years of age.

Despite the high prevalence and impact of sleep disturbance, training and education curricula for pediatric medical and allied health professions include little information or awareness building about sleep, particularly in strategies to ameliorate sleep for children and their families. An evident need to train health professionals working with children in evidence-based pediatric behavioral sleep care is emerging.

A recent partnership between Turkey and Australia has tackled this problem. By developing a scientific collaborative network between the two countries and using existing evidence-based methods, clinical information was shared between health professional workforces in these two countries as effective ways of translating knowledge in pediatric sleep interventions. Systematic training of primary and tertiary medical and allied health professionals must be considered as standard practice in tertiary education settings.

Keywords: Sleep, pediatric, insomnia

\section{INTRODUCTION}

The need for sleep education in the tertiary pediatric settings

Sleep is important for wellbeing. Sleep health is one of the major contributors to mental and physical health and as such should be an essential component of informed community and policy conversations around health. Increased conversations increase the potential for intervention. This is particularly the case in the first months and years of life in young families where sleep disturbance is common. In fact, it has been reported that sleep is the issue for which new parents seek the most help during the first years (1). Sleep problems, from either reduced sleep quantity or quality in young children, can be both significant and extensive and are not restricted to age, socioeconomic demographic or country (2). Non physiological sleep problems are commonly referred to as Behavioral Sleep Problems (BSP) and include difficulties initiating sleep alone, bedtime resistance, difficulties re-initiating sleep after normally occurring overnight waking (requiring parental assistance to re-settle) and/or early morning wakening (3). BSP affects up to $40 \%$ of children under two years of age and approximately $25 \%$ of children $2-10$ years old $(3,4)$.

\section{Clinical Research and Consequences}

What happens when parents do not get the assistance they need with sleep issues?

If healthy sleep habits are not well established in the early years, problems can persist in such a way that children can experience reduced sleep duration, concurrent or future behavioral problems, and academic difficulties (5). Furthermore, sleep disturbances in these age groups significantly impact sleep in the entire family and subsequently family function and mental and physical wellbeing of parents, particularly mothers (6). Many studies report causal relationships between sleep disturbed children and parental depression, anxiety and stress. 
Moreover, and importantly, when poor sleep is improved, related issues also improve (7).

So, early sleep education could be at the heart of improving firstly the sleep habits and then the general health of the community. It has been reported that the issue about which most help is sought by new parents concerns the sleeping habits of their child (1).

However, despite the high prevalence and impact of sleep disturbance, training and education curricula for pediatric medical and allied health professions include little information or awareness building about sleep, particularly in strategies to ameliorate sleep for children and their families. In Turkey, a quasi-experimental study found that $80 \%$ of pediatricians lacked basic skills and knowledge in behavioral pediatric sleep problems (8). The study also found that while a brief one-time training session improved their knowledge, there was still a need for more detailed training for skill acquisition. Indeed, a similar picture emerges in other countries and jurisdictions, where sleep training at all levels of training, (undergraduate, graduate or clinical training), is lacking $(9,10)$.

An evident need to train health professionals working with children in evidence-based pediatric behavioral sleep care is emerging.

\section{Types of sleep interventions available}

As these sleep disorders are considered behavioral in nature, the treatments utilized are also behavioral in nature (11). The most common behavioral sleep interventions are known as extinction methods as they "extinguish" the unwanted behavior of waking up overnight. All extinction methods involve the child's calling or crying being ignored once the child has been put to bed.

What happens when parents cannot access the sleep interventions they want?

Extinction methods can be considered to lie on a spectrum ranging from completely ignoring a child's nighttime protests or cries (an extinction method often called the "cry- it- outmethod"), to staying with the child until they fall asleep (often called the "camping out method") $(3,12)$. Between these is the graduated extinction method often called controlled crying or controlled comforting where parents periodically leave their child to cry in protest, checking less and less frequently (3). All of these treatments have been shown to achieve their aims of reducing nighttime crying and parental interaction by teaching parents to set limits on how much they will attend to their child and what they will do when they do attend their child $(3,12)$.

Given the lack of training in sleep for medical health professionals, the research literature is the first point of call for front line medical professionals to understand how to assist young children in improving their sleep. The research literature is overwhelmingly skewed towards extinction methods (12). Popular literature follows this trend with $61 \%$ (24 of 39 ) of parent sleep advice books endorsing extinction intervention (12). Hence when scanning the literature for behavioral sleep treatments, it is likely that the information presented and accessed by pediatric medical professionals is extinction based as these interventions are dominating the published literature. Therefore, it is likely that most medical health care professionals would be more likely to recommend extinction based interventions (12).

However, most standard extinction or graduated extinction treatments are often tolerated with difficulty by parents as the significant amount of crying (as the child communicates their desire for parental attention), can be difficult for a parent to withstand - particularly if they are instructed to ignore the cry $(13,14)$. Even in more gentle methods such as 'camping out' there are expectations that parents cannot comfort their child if distressed (5). It would appear then that if behavioral sleep treatments are to be successful, parents need to withstand and, in some cases, ignore their child's crying and this may not be tolerable for some parents. For these parents the treatment may be worse than the problem. On the other hand, there is emerging evidence to support the use of more responsive methods for those who find extinction approaches behaviorally or ideologically challenging (16). Behavioral sleep treatments that do not necessitate unattended crying are emerging and are often termed "responsive" methods because they do not ignore an infant's cries and may offer a less stressful option to parents attempting to improve their infant's sleep $(16,17)$.

So, the treatment of sleep in young children requires not only a significant understanding of the physiology of sleep health in these age groups, but also:

1. The expertise to be able to offer parents sleep inventions that combine efficacy with feasibility

2. Considers mother/infant attachment and stress levels of parents and child in the process

3. Reflects the different needs of parental preferences for intervention strategies.

A recent partnership between Turkey and Australia has tackled this problem.

By developing a scientific collaborative network between the two countries and using existing evidence-based methods, clinical information was shared between health professional workforces in these two countries as effective ways of translating knowledge in pediatric sleep interventions. The Turkish Social Pediatrics Association collaborated with an expert psychologist from Australia (Professor Sarah Blunden) specialized in pediatric behavioral sleep, to develop a course that combined didactic learning and practical implementation. A two-day course was run jointly by the Social Pediatrics Association and Blunden which provided 10hour long sessions for 35 health professionals. The training was available to pediatricians, nurses, and psychologists. The first part of the course was based on core competencies for 
health professional's training in pediatric behavioral sleep care (18). The second part covered responsive sleep interventions, and the implementation of a newly manualized responsive method of sleep intervention, The Gentle Method of Self Soothing (GeMSS) in particular (https://sensiblesleep.com/ course/blunden-responsive-gemss-method/). Pre and post questionnaire completion tested for knowledge acquisition while skill acquisition was assessed by post session case studies and clinical interactive discussions. Following the initial interactive face to face training, modules for GeMSS were provided online. A survey of 33 participants one year after completing the course found that $100 \%$ thought it was useful and $70 \%$ said that they had applied the techniques in their daily practice. Some suggested that learning together with colleagues, mentorship, and feedback cycles may be useful. Ten pediatricians were academics from universities who were able to offer training sessions to pediatricians, and residents in their own institutions, in this way, the training the trainer approach was implemented during the course as well. These educational efforts helped to raise awareness in pediatric behavioral sleep care. Conferences held by the Pediatric Societies still offer continuing professional development opportunities on pediatric behavioral sleep.

In pediatric settings therefore, opportunities arise to create the optimal environment for addressing sleep health issues due to the frequent and regular visits by families during the early years.

How the dissemination of sleep education can be incorporated into training opportunities for tertiary health care professionals, such as pediatricians and psychologists, in health sectors.

Training of health professionals in evidence-based pediatric behavioral sleep care employing similar methods as described above, will assist those working with children in a more systematic way. Furthermore, pediatric settings have the opportunity to deliver to parents and families specialist services that offer choices and options for parents to be able to improve the sleep of their children. Comprehensive training in pediatric behavioral sleep interventions could provide practitioners with an integrated well-targeted suite of clinical interventions (e.g. from extinction to responsive methods if required), that could cater for all parenting styles. Offering choice would potentially improve compliance, reduce attrition and ultimately benefit the sleep and wellbeing of all infants and their parents, especially those who struggle with traditional extinction methodologies (16).

The stepped care model (see below) of sleep information dissemination and intervention must reflect the differing levels of care and expertise needed and which should be available for families seeking assistance for sleep disturbance in their child.

Community organizations must be informed about sleep and sleep health offering basic sleep health knowledge and resources at a population level. This low-level information dissemination will act as a form of triage for those needing one on one assistance at a primary health level, potentially offering information through community organizations. The next level caters for those with some training in behavioral sleep medicine offering assistance to a more streamlined target population level and whose training has been overseen and is evidence based. Health professionals who need training in sleep include primary health care such as community doctors and nurses and general practitioners. The final level of sleep assistance reflects those specialized in sleep health and with mastery in sleep and specific organizations with expertise in how to improve sleep in children where sleep may be significantly disturbed and/or the child has complex needs. These tertiary level health professionals would include sleep psychologists, sleep physicians and pediatricians.

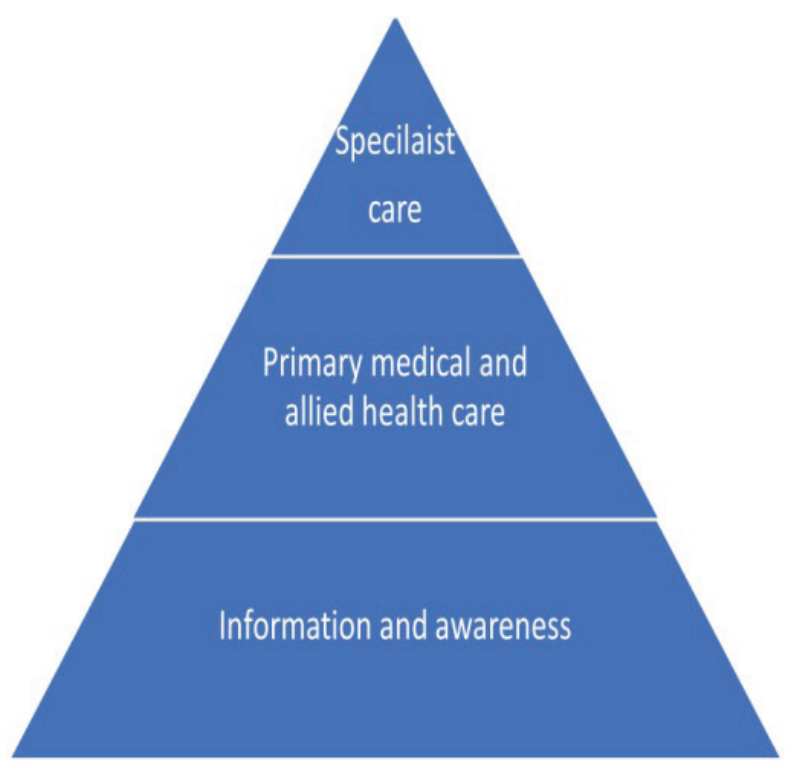

Figure 1. Stepped care model of sleep health needs

\section{RECOMMENDATIONS}

1. Increase sleep education in undergraduate and post graduate medical and allied health training. This requires some substantial changes to curricula and policy.

2. In the absence of increased sleep content curricula, in the short term, there should be training of medical and allied health professionals to address sleep health issues. Training of the pediatric workforce should be through:

a. Developing relevant resources

b. Extra curricula and post graduate training in sleep to professionals already in the field through private organizations or public institutions Training must be evidence based and include mastery testing and include competency-based assessment.

c. Implementation of ongoing professional development in sleep education

d. Establishment of sleep communities of practice for professionals across jurisdictions

e. Attendance at and organization of cross-discipline conferences. Sleep content should be included in 
pediatric congresses and conference meetings of medical and allied health professionals as part of a holistic approach to pediatric health

f. Ensuring families are offered sleep interventions that correspond to the intervention of their choice at primary and tertiary level interventions.

\section{CONCLUSION}

Sleep health is one of the major contributors to mental and physical health and as such should be an essential component of informed community and policy conversations around health.

Systematic training of primary and tertiary medical and allied health professionals must be considered as standard practice in tertiary education settings.

Hakem Değerlendirmesi: Dış bağımsız.

Yazar Katkıları: Çalışma Konsepti/Tasarım- S.B., P.B.; Yazı Taslağı- S.B., P.B.; Son Onay ve Sorumluluk- S.B., P.B.

Çıkar Çatışması: Yazarlar çıkar çatışması beyan etmemişlerdir.

Finansal Destek: Yazarlar finansal destek beyan etmemişlerdir.

Peer Review: Externally peer-reviewed.

Author Contributions: Conception/Design of Study- S.B., P.B.; Drafting Manuscript- S.B., P.B.; Final Approval and Accountability- S.B., P.B.

Conflict of Interest: Authors declared no conflict of interest.

Financial Disclosure: Authors declared no financial support.

\section{REFERENCES}

1. Newton AT, Corkum PV, Blunden S, Reid GJ. Influences on helpseeking decisions for behavioral child sleep problems: Why parents do and do not seek help. Clinical Child Psychology and Psychiatry 2021;26(1):207-21. doi: 10.1177/1359104520963375

2. Kim DS, Lee $\mathrm{CL}$, Ahn YM. Sleep problems in children and adolescents at pediatric clinics. Korean J Pediatr 2017;60(5):15865. doi: $10.3345 / \mathrm{kjp} .2017 .60 .5 .158$

3. Meltzer LJ, Mindell JA. Systematic Review and Meta-Analysis of Behavioral Interventions for Pediatric Insomnia. J Pediatr Psychol 2014;39(8):932-48. doi: 10.1093/jpepsy/jsu041.

4. Simola P, Laitalainen E, Liukkonen K, Virkkula P, Kirjavainen T, Pitkaranta A, et al. Sleep disturbances in a community sample from preschool to school age. Child Care Health Dev 2012;38(4):572-80. doi: 10.1111/j.1365-2214.2011.01288.x.
5. Gregory AM, O'Connor TG. Sleep problems in childhood: A longitudinal study of developmental change and association with behavioral problems. J Am Acad Child Adolesc Psychiatry 2002;41(8):964-71.doi:10.1097/00004583-200208000-00015.

6. Hiscock H, Bayer JK, Hampton, Ukoumunne OC, Wake M. Longterm mother and child mental health effects of a population-based infant sleep intervention: cluster-randomized, controlled trial. Pediatrics 2008;122(3):e621-e627.doi: 10.1542/peds.2007-3783.

7. Williamson AA, Mindell JA, Hiscock H, Quach J. Longitudinal sleep problem trajectories are associated with multiple impairments in child well-being. J Child Psychol Psychiatry 2020;61(10):1092-103. doi: $10.1111 /$ jcpp.13303

8. Ersu R, Boran P, Akın Y, Bozaykut A, Ay P, Yazar AS. Effectiveness of a sleep education program for pediatricians. Pediatr Int 2017;59(3):280-5.

9. Gruber R, Constantin E, Frappier JY, Brouillette RT, Wise MS. Training, knowledge, attitudes and practices of Canadian health care providers regarding sleep and sleep disorders in children. Paediatric Child Health 2017;22(6):322-27.doi: 10.1093/pch/pxx069

10. Meaklim H, Jackson ML., Bartlett D, Saini B, Falloon K, Junge M, et al. Sleep education for healthcare providers: addressing deficient sleep in Australia and New Zealand. Sleep Health 2020;6(5):63650. doi: 10.1016/j.sleh.2020.01.012.

11. Hill C. Practitioner review: effective treatment of behavioural insomnia in children. J Child Psychol Psychiatry 2011;52(7):73140. doi: 10.1111/j.1469-7610.2011.02396.x

12. Etherton $\mathrm{H}$, Blunden $\mathrm{S}$, Hauck Y. Discussion of ExtinctionBased Behavioral Sleep Interventions for Young Children and Reasons Why Parents May Find Them Difficult. J Clin Sleep Med 2016;12(11):1535-43. doi: 10.5664/jcsm.6284.

13. France KG. Handling parents' concerns regarding the behavioural treatment of infant sleep disturbance. Behaviour Change 1994;11(2):101-9. doi:10.1017/S0813483900004617

14. Blunden S, Etherton H, Hauck Y. (2016). Resistance to cry intensive sleep interventions in young children: Are we ignoring children's cries or parental concerns? Children 2016;3(2):8. doi:10.3390/ children3020008

15. Ferber R. Solve your child's sleep problems. 1998. New York, Fireside Publications.

16. Blunden $S$, Dawson D. Behavioural sleep interventions in infants: Plan B - Combining models of responsiveness to increase parental choice. Journal of Paediatrics and Child Health 2020;56:675-79. https://doi.org/10.1111/jpc.14818

17. Ozturk M, Boran P, Ersu R, Peker Y. Possums-based parental education for infant sleep: cued care resulting in sustained breastfeeding. Eur J Pediatr 2021;180(6):1769-76. doi: 10.1007/ s00431-021-03942-2.

18. Boerner KE, Coulombe JA, Corkum P. Core competencies for health professionals' training in pediatric behavioral sleep care: a Delphi Study. Behavioral Sleep Med 2015;13(4):265-84. doi: 10.1080/15402002.2013.874348. 